\title{
Nahm's Transformation on the Lattice
}

\author{
A. González-Arroyo* and C. Pena \\ Departamento de Física Teórica $C$-XI \\ Universidad Autónoma de Madrid \\ Cantoblanco, Madrid 28049, SPAIN.
}

\begin{abstract}
By studying zero modes of the Dirac operator on the lattice, we explicitly construct the Nahm transform of some topologically non-trivial gauge field configurations on the torus.
\end{abstract}

KEYWORDs: Nahm transform, Fermion zero modes, Instanton solutions, Lattice

gauge theory.

*also at: Instituto de Física Teórica C-XVI, Universidad Autónoma de Madrid, Cantoblanco, Madrid 28049, SPAIN. 


\section{Contents}

1. Introduction 1

2. The Nahm transformation 3

3. Solving the homogeneous and inhomogenous Dirac equation on the lattice 6

4. Application to the case of non-trivial $S U(2)$ gauge field configurations on the torus 8

5. Conclusions and future outlook 13

\section{Introduction}

Classical Yang-Mills fields have been the subject of intense study in the literature. Besides being interesting objects in their own right, much of the motivation arises from the relevance of non-abelian gauge theories for our understanding of the interactions among elementary particles. Although, in that case, one is concerned with quantum fields, particular classical configurations have been argued to play a fundamental role in explaining some phenomena. In this context, the pioneer work of Polyakov [1] showed how pseudoparticle solutions become relevant in the semiclassical approximation to the path integral. These solutions are then argued to be responsible of some of the most intriguing effects of Quantum Field Theory. This is the case for the Confinement property of the 3-dimensional compact abelian model, explained originally by Polyakov. In 4 dimensions 't Hooft [2] showed that instantons [3] provide an explanation for the so-called $U(1)$-problem. This triggered a joint effort of physicists and mathematicians in the 70's, which culminated with the construction of all self-dual solutions to the euclidean Yang-Mills equations compactified on a sphere [4]. On physical grounds, the compactification on a sphere is a condition equivalent to the requirement of finite classical action. In other words, one is looking for solutions which exist isolated, i.e. surrounded by the perturbative vacuum $\left(F_{\mu \nu}=0\right)$. However, in Quantum Field Theory neither the requirement of finite action nor the one of isolated solutions is compulsory. For example, it was suggested by the work of Saviddy [5] and the Copenhagen group [6] that the QCD vacuum was permeated by some structures carrying chromo-magnetic flux. These are neither isolated nor carry finite action. Henceforth, it could also be relevant and interesting to study classical solutions with different boundary conditions. 
In this respect a good deal of interest has focused on the study of Yang-Mills fields on the 4-dimensional torus (for a review see Ref. [7]). The torus adds topological features which have an appealing physical interpretation [8]. Furthermore, solutions on the torus can be considered solutions on $\mathbf{R}^{4}$ which are periodic, with a total action which diverges (with finite action per unit cell). Unfortunately, despite the effort, only very special self-dual solutions on the torus, having constant field strength, are known analytically [9, 10]. The lattice formulation of Yang-Mills theory has proven a precise and efficient method for obtaining these solutions numerically [11, 12]. A basic building block of many of these configurations is a certain lump carrying fractional topological charge $Q=\frac{1}{N}$. These lumps cannot exist isolated - their size is determined by the distance to their neighbours. The moduli space of a self-dual solution on the torus, given by the index theorem, has four parameters per lump, which can be associated to the coordinates of its center. This suggests that one can obtain self-dual solutions on $\mathbf{R}^{4}$ by deforming these configurations away from the periodic arrangement (at no cost of action). A picture of the Yang-Mills quantum vacuum as a liquid of these fractional topological charge lumps, as suggested by our group [13, could account at the same time for the observed string tension and topological susceptibility.

On the analytical side, an interesting tool is provided by the Nahm transformation [14]. This transformation maps a $U(N)$ self-dual gauge field on the torus with topological charge $Q$ into a $U(Q)$ self-dual gauge field on the dual torus with topological charge $N$. The use of this transformation, which can be considered a particular case of a duality transformation, allows one to prove that, in the absence of twist, there are no $Q=1$ instanton solutions on the torus (see for example 15]). Its use has proved useful as well for constructing new instanton solutions in $S_{1} \times \mathbf{R}^{3}$ [16]. For the torus case, it has been shown that the constant field strength solutions in $S U(2)$ are mapped into themselves [17]. In this paper we will show that it is possible to use lattice techniques to explicitly construct the Nahm transform of a given self-dual lattice gauge field configuration. This opens the door to the possibility of systematically investigating the properties of the Nahm transformation for the whole set of self-dual gauge field configurations. In the paper we will apply the method to the case of one of the non-trivial self-dual solution on the torus, producing accurate results for its transform. As a matter of fact, this together with the study of the zero modes for the Dirac equation in the adjoint representation, which are supersymmetric partners of the gauge field, provides new numerical descriptions of the torus self-dual gauge fields, with some relative advantages over the conventional discretization. There is also hope that, as in the aforementioned $S_{1} \times \mathbf{R}^{3}$ case, this study could eventually lead to an analytical approach to these classical fields.

The layout of the paper is as follows. In the next section, we present the details of the Nahm transformation and the main formulas which relate our problem to the solution of homogeneous and inhomogeneous Dirac equations. In the following section, we explain how one can formulate the latter on the lattice, and describe the numerical 
techniques that we use to solve them. In section 4, we apply the technique to the actual construction of the Nahm transform for some input self-dual configurations. We first test the method by looking at its result in a known case, and then apply it to other non-trivial self-dual configuration. Finally, in section 5 we summarize our results and discuss future prospects.

\section{The Nahm transformation}

Let us consider a 4 dimensional torus of size $l_{0} \times l_{1} \times l_{2} \times l_{3}$, and let $\hat{l}_{\mu}$ represent the vector $\left(0, \ldots, l_{\mu}, \ldots, 0\right)$, whose only non-zero component is the $\mu^{\text {th }}$ component. Now consider a self-dual gauge field configuration $A_{\mu}(x)$ defined on this torus. It satisfies:

$$
A_{\nu}\left(x+\hat{l}_{\mu}\right)=\Omega_{\mu}(x) A_{\nu}(x) \Omega_{\mu}^{+}(x)+\imath \Omega_{\mu}(x) \partial_{\nu} \Omega_{\mu}^{+}(x)
$$

where $\Omega_{\mu}(x)$ are the twist matrices. For $S U(N)$, these matrices must fulfill the consistency condition:

$$
\Omega_{\mu}(x+\nu) \Omega_{\nu}(x)=Z_{\mu \nu} \Omega_{\nu}(x+\mu) \Omega_{\mu}(x)
$$

When fields transforming in the fundamental representation of the gauge group appear, all the constants $Z_{\mu \nu}$ must be equal to 1 ; otherwise, they are in general elements of the center of the group $\mathbf{Z}_{N}$, which can be parametrized as $Z_{\mu \nu}=\exp \left(2 \pi \imath n_{\mu \nu} / N\right)$. The twist tensor $n_{\mu \nu}$ is antisymmetric, and its elements are integers defined modulo $N$.

Let $Q$ stand for the topological charge of the gauge field configuration. As is wellknown, the Atiyah-Singer index theorem implies that the difference between the number of positive chirality and negative chirality solutions of the Dirac equation for fermion fields transforming in the fundamental representation of the gauge group is given by $Q$. Let us assume that for our gauge configuration there are no negative chirality solutions. Then, there are exactly $Q$ positive chirality solutions, which we will label $\Psi^{\alpha}(x)$, with $\alpha=1, \ldots, Q$. They satisfy:

$$
\hat{\bar{D}} \Psi^{\alpha}(x)=0
$$

where $\hat{\bar{D}} \equiv D_{\mu} \bar{\Gamma}_{\mu}$ is the positive chirality Weyl operator and $D_{\mu}=\partial_{\mu}-\imath A_{\mu}$. In the Weyl basis we have:

$$
\not D=\left(\begin{array}{cc}
0 & \hat{D} \\
\hat{\bar{D}} & 0
\end{array}\right) \quad \gamma_{5}=\left(\begin{array}{cc}
1 & 0 \\
0 & -1
\end{array}\right)
$$

where $\Gamma_{\mu}=(I,-\imath \vec{\sigma})$ and $\bar{\Gamma}_{\mu}=\Gamma_{\mu}^{+}$. Furthermore, the solutions satisfy the following boundary conditions:

$$
\Psi^{\alpha}\left(x+\hat{l}_{\mu}\right)=\Omega_{\mu}(x) \Psi^{\alpha}(x) .
$$

Now consider the family of gauge fields:

$$
A_{\mu}(x, z)=A_{\mu}(x)+2 \pi z_{\mu} I
$$


where $z_{\mu}$ are 4 real numbers. For all $z$, the field strength $F_{\mu \nu}$ is the same, and hence they are all self-dual and have the same topological charge. Therefore, we obtain a family $\Psi^{\alpha}(x, z)$ of positive chirality solutions of the Dirac equation:

$$
\hat{\bar{D}}_{z} \Psi^{\alpha}(x, z)=(\hat{\bar{D}}-2 \pi \imath \hat{\bar{z}} I) \Psi^{\alpha}(x, z)=0
$$

satisfying the boundary condition Eq. 2.5 and normalized as follows:

$$
\int d^{4} x\left(\Psi^{\alpha}(x, z)\right)^{+} \Psi^{\beta}(x, z)=\delta_{\alpha \beta}
$$

Now notice that $\exp \left(-2 \pi \imath \tilde{z}_{\mu} x_{\mu}\right) \Psi^{\alpha}(x, z+\tilde{z})$ satisfies the same equation than $\Psi^{\alpha}(x, z)$. However, in general, the boundary conditions are different, since the right-hand side of Eq. 2.5 gets multiplied by $\exp \left(-2 \pi \imath \tilde{z}_{\mu} l_{\mu}\right)$. This new factor becomes simply unity if $\tilde{z}_{\mu}$ is an integer multiple of $1 / l_{\mu}$. Hence, defining the vector $\hat{\tilde{l}}_{\mu}=\left(0, \ldots, \frac{1}{l_{\mu}}, \ldots, 0\right)$ we can write:

$$
\Psi^{\alpha}\left(x, z+\hat{\tilde{l}}_{\mu}\right)=\Psi^{\beta}(x, z)\left(\Omega_{\mu}^{\prime+}(z)\right)_{\beta \alpha} \exp \left(2 \pi \imath x_{\mu} / l_{\mu}\right)
$$

This is so because any solution can be written as a linear combination of the basis functions $\Psi^{\beta}(x, z)$. The coefficients $\left(\Omega_{\mu}^{\prime+}(z)\right)_{\beta \alpha}$ cannot in general be chosen equal to 1 , if we insist in $\Psi^{\beta}(x, z)$ being continuous in $z$.

Now let us construct the Nahm transform of the gauge field $A_{\mu}(x)$. It is given by:

$$
\left(\hat{A}_{\mu}(z)\right)_{\alpha \beta}=\imath \int d^{4} x\left(\Psi^{\alpha}(x, z)\right)^{+} \frac{\partial}{\partial z_{\mu}} \Psi^{\beta}(x, z) .
$$

This is a $U(Q)$ gauge field defined on the dual torus (of size $\frac{1}{l_{0}} \times \frac{1}{l_{1}} \times \frac{1}{l_{2}} \times \frac{1}{l_{3}}$ ). Using Eq. 2.9 one finds that $\hat{A}_{\mu}(z)$ satisfies a relation analogous to Eq. 2.1 (exchanging the roles of $\mathrm{x}$ and $\mathrm{z}$ ) in terms of $\Omega_{\mu}^{\prime}$. Now, one can in terms of this field construct the field strength tensor $\hat{F}_{\mu \nu}$. The Nahm-transformed gauge field has the following properties:

- $\hat{F}_{\mu \nu}$ is again self-dual.

- The first and second Chern classes and the ranks for the original and transformed gauge fields are related through:

$$
\begin{aligned}
& r k(\hat{F})=c_{2}(F)-\frac{1}{2} c_{1}^{2}(F) \\
& c_{1}(\hat{F})=-\int_{T^{4}}\left(d z_{\mu} \wedge d x_{\mu}\right)^{2} \wedge c_{1}(F) \\
& c_{2}(\hat{F})=r k(F)+\frac{1}{2} c_{1}^{2}(F)
\end{aligned}
$$

Thus, in particular, one sees that the roles of the rank of the group and the topological charge are exchanged by the Nahm transformation when the first Chern class vanishes. 
- From the previous statement it follows that if we start with $S U(N)$ gauge fields (with no twist $n_{\mu \nu}=0 \bmod N$ ), then the Nahm transform is in $S U(Q)$.

- The Nahm transformation is an involution: if we apply it twice we go back to the original gauge field. Thus, it can be considered a duality transformation.

- If we start with a family of gauge fields depending on some parameters, then the Nahm transformation generates a new set of self-dual gauge fields depending on those parameters. Hence, we have induced a mapping between the moduli spaces of the gauge field and its transform. This mapping is an isometry with respect to the natural metric of these moduli spaces.

For a proof of these properties, see Ref. [15].

Now, let us consider the vicinity of a point $z$ in the dual torus. We can make a Taylor expansion of the positive chirality solutions of the Dirac equation in the vicinity of this point:

$$
\Psi^{\alpha}(x, z+\Delta z)=\Psi^{\alpha}(x, z)+\Delta z_{\mu} \Psi_{\mu}^{\alpha}(x, z)+\Delta z_{\mu} \Delta z_{\nu} \Psi_{\mu \nu}^{\alpha}(x, z)+\ldots
$$

By plugging this equation into the Dirac equation for $z+\Delta z$ and equating powers of $\Delta z_{\mu}$ on both sides, we obtain for the first two orders:

$$
\begin{aligned}
& \hat{\bar{D}}_{z} \Psi^{\alpha}(x, z)=0 \\
& \hat{\bar{D}}_{z} \Psi_{\mu}^{\alpha}(x, z)=2 \pi \imath \bar{\Gamma}_{\mu} \Psi^{\alpha}(x, z)
\end{aligned}
$$

Now, in terms of these functions, and defining:

$$
\begin{aligned}
& P_{\mu}^{\alpha \beta}(z) \equiv\left\langle\Psi^{\alpha} \mid \Psi_{\mu}^{\beta}\right\rangle \equiv \int d^{4} x\left(\Psi^{\alpha}(x, z)\right)^{+} \Psi_{\mu}^{\beta}(x, z) \\
& Q_{\mu \nu}^{\alpha \beta}(z) \equiv\left\langle\Psi_{\mu}^{\alpha} \mid \Psi_{\nu}^{\beta}\right\rangle
\end{aligned}
$$

one can write the vector potential and the field-strength tensor coming out of the Nahm transformation as follows:

$$
\begin{aligned}
\hat{A}_{\mu}(z) & =\imath P_{\mu}(z) \\
\hat{F}_{\mu \nu}(z) & =\imath\left(Q_{\mu \nu}(z)-Q_{\mu \nu}^{+}(z)+\left[P_{\mu}(z), P_{\nu}(z)\right]\right)
\end{aligned}
$$

The normalization conditions imply that $\hat{A}_{\mu}(z)$ is hermitian. Henceforth, to obtain the Nahm-transformed gauge field at one point $z$ of the dual torus, one has simply to solve Eqs. 2.15-2.16. None of the two equations has a unique solution. Choosing a solution within both sets of equations amounts to a choice of gauge for the Nahm-transformed fields. More specifically, any normalized set of solutions of Eq. 2.15 is related by a unitary transformation $\Omega_{\alpha \beta}^{\prime}(z)$ to any other. Furthermore, once this choice is made, it can be easily shown that selecting a particular solution $\Psi_{\mu}^{\alpha}(x, z)$ of Eq. 2.16 amounts to the choice of a gauge in the neighbourhood of $z$. One can obtain any solution of 
Eq. 2.16 by adding to $\Psi_{\mu}^{\alpha}(x, z)$ a general solution of Eq. 2.15: $\Psi_{\mu}^{\alpha}(x, z)+S_{\mu}^{\beta \alpha} \Psi^{\beta}(x, z)$, where $S_{\mu}$ must be antihermitian due to the normalization conditions. This produces a change in the vector potential $\hat{A}_{\mu} \longrightarrow \hat{A}_{\mu}+\imath S_{\mu}$, but as can be readily verified from the equations above, $\hat{F}_{\mu \nu}$ is left invariant. It is in principle possible to impose some set of conditions on the solutions in order to select a particular gauge for $\hat{A}_{\mu}$. However, in this paper we will concentrate on gauge invariant quantities and, hence, any solution will do.

In the following sections we will describe how we have been able to numerically construct the Nahm transform of a given self-dual gauge field configuration on the torus by finding the solutions of Eqs. 2.15, 2.16 using the lattice formulation of the theory. In the next section we will describe the numerical technique and in the following we will apply our construction to some explicit examples.

\section{Solving the homogeneous and inhomogenous Dirac equation on the lattice}

In this section we will explain how we can actually obtain the solutions of Eqs. 2.15 2.16 by studying the problem on the lattice.

All studies of the Dirac equation on the lattice have to deal with the well-known problem of fermion doubling. This usually means that one must either break chiral invariance explicitly by using Wilson fermions or maintain it at the expense of producing spureous lattice solutions to the equation. A middle way is represented by the use of staggered fermions. To put Dirac equations on the lattice we have used both the Wilson action, keeping the value of the Wilson parameter $r$ free, and a naive fermion action; in both cases, we set the lattice fermion mass equal to zero. The second case allows for Weyl (2-component) spinors, while the first one requires Dirac (4-component) spinors. The results we report here have been obtained with Wilson fermions, so we restrict our discussion to this latter case.

Our Wilson-Dirac operator reads (the superscript $L$ stands for lattice):

$$
\begin{aligned}
& \not D^{L} \psi(n)=4 r \psi(n)-\frac{1}{2} \sum_{\mu}[\left(r-\gamma_{\mu}\right) U_{\mu}(n) \psi(n+\hat{\mu})+ \\
&\left.+\left(r+\gamma_{\mu}\right) U_{\mu}^{+}(n-\hat{\mu}) \psi(n-\hat{\mu})\right],
\end{aligned}
$$

where $U_{\mu}$ is the gauge field, $\hat{\mu}$ is a unitary lattice vector in the $\mu$ direction, and $\gamma_{\mu}$ are Dirac matrices. To add a constant $U(1)$ potential, as required by the construction of the Nahm transformation, we exponentiate the whole $A_{\mu}+2 \pi z_{\mu} I$ to build link variables. Thus, $\not z_{z}^{L}$ has the same form as above but with $U_{\mu}$ replaced by $\exp \left(-2 \pi \imath a z_{\mu}\right) U_{\mu}$, where $a$ is the lattice spacing. Note that the argument of the exponential is conveniently dimensionless, as the coordinates $z_{\mu}$ in the dual space have dimensions of inverse length.

Our first goal is to solve numerically homogeneous Dirac equations in a given background lattice gauge configuration. For this purpose, we construct the hermitian positive operator $\left(\gamma_{5} \not D^{L}\right)^{2}$, for which we expect to identify a lowest eigenspace of smooth 
modes, whose degeneracy must be equal to the one given by the index theorem. The elements of this space can be identified as a lattice approximation to the continuum zero modes. Although in general no exact fermionic zero modes will live in the lattice, the lowest eigenvalue of $\left(\gamma_{5} \not D^{L}\right)^{2}$ must approach zero with finer discretization to signal the recovery of continuum behaviour. All this requirements are indeed verified by our results, and thus we translate the problem of solving homogeneous Dirac equations into that of finding out these lowest eigenvectors.

Other problem we face when computing solutions is that of keeping the right chirality for the fermion fields. Our technique to achieve this proceeds by tuning the value of $r$. There is a convenient window within the interval $[0,1]$ in which $r$ is large enough to push up the doublers, and small enough so as to maintain approximate chirality for the resulting solutions. This range of values can be determined by computing with different $r$, then checking the properties of the result with different criteria [18, 19. A typical value in our computations is $r=10^{-2}$.

We are finally able to obtain vectors that are approximately chiral, the "wrong" half (chirality opposite to the one dictated by the sign of the topological charge of the gauge configuration) of the spinor being numerically negligible everywhere with respect to the "right" half. Above the lowest space, whose degeneracy is given by the index theorem, the typical structure for the lowest part of our spectra presents a first excited continuum level, the order of magnitude of its eigenvalue being stable with changing lattice volume, and a set of doubler states between this excited level and the zero modes level. We can recognize the doublers by the oscillating behaviour of their invariant densities, as well as by comparison with the known doubling symmetry pattern. Moreover, the evolution with $r$ of the respective eigenvalues for the quasi-zero modes and the doublers is qualitatively different. The gap between the approximate zero modes level and the doubler levels is determined essentially by the value of $r$.

To compute lowest eigenvectors of the operator $\left(\gamma_{5} \not D^{L}\right)^{2}$ we have used a standard conjugate gradient ( $\mathrm{CG}$ ) method, basically the one described in [20] (without implementing the acceleration method described in this reference), which gives eigenvectors of the hermitian, positive definite operator $A$ with increasing eigenvalue by minimizing the Ritz functional:

$$
\mu(v)=\frac{<v, A v>}{<v, v>}
$$

with $v$ a trial $\mathrm{CG}$ vector.

To check by consistency our CG method we have performed alternative calculations using both a Lanczos procedure and a minimization technique inspired by the cooling algorithm used in lattice Yang-Mills studies. The results for the zero modes in a number of qualitatively different gauge configurations will be the subject of future publication [19].

To solve inhomogenous Dirac equations of the form $\not D \psi=\phi$, as required by our construction of the Nahm transformation through a local Taylor expansion, we use a variant of the CG method, the stabilized biconjugate gradient algorithm (BiCGStab) [21], 
whose adaptation for the purpose of inverting lattice Dirac operators is described in [22]. We have used directly the implementation described in this latter reference, with minor operative changes. The basic idea of the construction is the following: if we apply CG to an inhomogenous equation of the form $A v=w$, for each iteration we will have a trial solution $v_{i}$, a search direction $p_{i}$ and a residue $r_{i}=w-A v_{i}$; the new trial vector is constructed as $v_{i+1}=v_{i}+\alpha p_{i}$, with $\alpha$ determined by the requirement that the new residue has minimum norm, and the problem is to find the new $p_{i+1}$ (which fulfills the same condition for search directions as in "raw" $\mathrm{CG}$ ) and $r_{i+1}$ with the lowest computational cost, which essentially means with a lowest number of operator-vector multiplications. The BiCG method incorporates an additional sequence of vectors $\hat{r}_{i}$ such that $r_{j}^{+} \hat{r}_{i}=\hat{r}_{j}^{+} r_{i}=0$ for $i=0,1, \ldots, j-1$. With this construction, the residuals satisfy

$$
r_{j}=P_{j}(A) r_{0}, \hat{r}_{j}=P_{j}\left(A^{+}\right) \hat{r}_{0}
$$

where $P_{j}$ are polynomials of degree $\leq j$. If the method converges, these polynomials can be identified as 'reductors' for the norm of the residue.

This convergence behaviour can be enhanced as in the conjugate gradient squared (CGS) algorithm, which furthermore avoids the computation of the $\hat{r}_{i}$ and makes residuals satisfy $r_{j}=P_{j}^{2}(A) r_{0}$, with the same $P_{j}$. However, the convergence of CGS is usually far from being smooth, and the possibility exists of obtaining large peaks for $\left|r_{i}\right|$ which can plague a systematic application of the method. The BiCGStab cures this problem by making $r_{j}=Q_{j}(A) P_{j}(A) r_{0}$, with an appropriate form for the polynomial $Q_{j}$ so as to keep a good reduction behaviour while keeping in a smoother regime the evolution of $\left|r_{i}\right|$. Further technical details can be found in the references.

The application of this method to our problem allows us to efficiently compute approximate solutions, with typical values for $|\phi-\not D \psi|$ far below the corrections to continuum quantities eventually coming from other sources, thus not adding significative new errors to the calculations in which these vectors enter.

\section{Application to the case of non-trivial $S U(2)$ gauge field con- figurations on the torus}

In order to test the applicability and stability of our method we have first studied a case for which an explicit analytical solution is known. This occurs for gauge configurations with constant field strength. The Dirac equation in this background can be solved by techniques which are very similar to the ones used to study small deformations around these gauge fields [10]. An explicit example has been worked out in Ref. [23]. For comparison we take precisely this case in our numerical work.

Our gauge configuration has twist tensor defined by $n_{03}=n_{21}=2$, the other components being zero; its topological charge is 2 . The gauge potential and the field 
strength tensor can be written as:

$$
\begin{aligned}
A_{\mu} & =-\frac{1}{2} \pi \frac{n_{\mu \nu}}{l_{\mu} l_{\nu}} x_{\nu} \sigma_{3} \\
F_{\mu \nu} & =\pi \frac{n_{\mu \nu}}{l_{\mu} l_{\nu}} \sigma_{3}
\end{aligned}
$$

where $l_{\mu}$ are, as defined before, the lengths of the torus, which we set equal to 1 . (Note that this $F$ is actually anti-self-dual). The Nahm transformation can be carried out analytically in this case [23], and the result is $\hat{A}_{\mu}=-A_{\mu}, \hat{F}_{\mu \nu}=\tilde{F}_{\mu \nu}=-F_{\mu \nu}$ with the natural gauge choice for the transformed fields.

To compare with this result, we first put the configuration on the lattice, which can be done exactly by exponentiating the above form for $A_{\mu}$ to build links. Now, the required numerical solutions to lattice Dirac equations can be computed, and the transformed field extracted from their scalar products according to our procedure. To be able to obtain a large set of transformed points, we have worked in a $8^{4}$ lattice. At the end we get a perfect structural reproduction of the exact results, with only a slight error for the field value. The only nonvanishing fields for our numerical transformation are $E_{3}$ and $B_{3}$, as required, and self-duality is verified almost exactly within the machine numerical precision; when a gauge is fixed (by directly diagonalizing the fields at each $z$-point, because the gauge fixing procedure we describe below for nontrivial configurations does not make sense in this case), we end up with:

$$
E_{3}=B_{3}=1.05 E_{3}^{C}
$$

with $E_{3}^{C}$ the continuum field. The $5 \%$ difference must come from the discretization corrections to the solutions of Dirac equations, which are sizable due to the small number of lattice points. In any case, we can conclude that our numerical implementation of the Nahm transformation is working satisfactorily for this test example.

Now let us consider another case for which no analytical solution is known. Motivated by our own interest in this type of configurations, our starting point is a self-dual $S U(2)$ gauge field configuration with non-trivial twist and fractional topological charge. In particular, we take a solution having non-orthogonal twist given by the twist tensor:

$$
n_{0 i}=(1,0,0) \quad \frac{1}{2} \epsilon_{i j k} n_{j k}=(1,1,1)
$$

Given this twist, the topological charge must be a half integer value, equal to the action divided by $8 \pi^{2}$. We are interested in the absolute minimum action solution in this twist sector. This has action $4 \pi^{2}$ and topological charge $\frac{1}{2}$. A configuration of this type, which is unique modulo space-time translations and discrete symmetries, is easy to obtain numerically by minimizing the lattice action in this twist sector (see, for example, Refs. [11, 12]). The action density consists on a single lump with size of 
the order of the smallest torus length. For reasons that will be clear soon we took the torus sizes in the ratio $l_{0}: l_{1}: l_{2}: l_{3}=2: 1: 2: 1$.

However, $S U(N)$ configurations with twist are not good starting points for the Nahm transformation since these boundary conditions are singular for spinors transforming in the fundamental representation. There is a number of ways out of this problem. We will choose the following one. Instead of considering a single torus period we will consider more than one period in the short directions. The resulting configuration, after applying an appropriate gauge transformation, has no twist or singularity in the new torus of size $l_{\mu}=1$. Gauge invariant quantities are periodic with period $\frac{l_{\mu}}{2}$ in the 1 and 3 directions. Hence the action density consists on four lumps like those described previously and the total topological charge and action are 2 and $16 \pi^{2}$ respectively. In Fig. 1 we plot the action density in the $x_{1}-x_{3}$ plane setting the other two coordinates to the value giving maximal action density. This is the original gauge field configuration to which we will apply the Nahm transformation. This will again be a self-dual $S U(2)$ configuration with topological charge equal to 2.

In the numerical construction of the $Q=1 / 2$ lump we have worked in a lattice of size $12 \times 6 \times 12 \times 6$. After "glueing" the lump to itself to obtain the configuration explained above, the result is a $12^{4}$ lattice, where all the subsequent computations are carried out.

The first thing we did was to look at the solutions of the Dirac equation in the background of this configuration. Indeed on the lattice there are no exact solutions. What we actually did, as explained in the previous chapter, was to find the smallest eigenvalue of the operator $\left(\gamma_{5} \not D^{L}\right)^{2}$ and the corresponding eigenvectors. There are 2 linearly independent degenerate solutions. We will choose the following procedure to choose a basis in this two-dimensional space (which fixes the gauge for $\hat{F}$ ). Consider one of the points $x_{0}$ on the lattice where the action density peaks (there are 4 points of this type). Now we choose the basis of solutions of the Dirac equation such that the hermitian matrix $M_{\alpha \beta}\left(x_{0}\right)$ is diagonal:

$$
M_{\alpha \beta}\left(x_{0}\right) \equiv\left(\Psi^{\alpha}\left(x_{0}\right)\right)^{+} \Psi^{\beta}\left(x_{0}\right)=\left(\begin{array}{cc}
\lambda_{1} & 0 \\
0 & \lambda_{2}
\end{array}\right) .
$$

It is a scalar product (sum over spin and color indices) of the $2 N$-dimensional vectors $\Psi^{\alpha}\left(x_{0}\right)$. We choose $\lambda_{1}>\lambda_{2}$. This condition does not fix completely the solutions, one is still free to multiply each element of the basis by an independent phase. We fix the remaining arbitrarity by imposing $M_{\alpha \beta}\left(x_{0}^{\prime}\right)$ to be real, where $x_{0}^{\prime}=x_{0}+\frac{1}{2} \hat{l}_{1}$ is another of the points where the action density peaks. There is an overall choice of phase which will not affect our results. Our first result will be to give the density matrix $M_{\alpha \beta}(x)$, which is a (gauge invariant with respect to transformations of the original gauge field) $2 \times 2$ hermitian matrix field defined on the original torus. In Fig. 2 we show the value of the corresponding densities $M_{11}$ and $M_{22}$ in the same plane where we gave the action density of the configuration before. Notice that the first solution peaks precisely at a point $x_{0}$ where the action density peaks, and was used to fix a basis in the space 
of solutions. On the contrary the density of the other solution $M_{22}$ shows the same behaviour but displaced by a vector $\left(0, \frac{1}{2}, 0, \frac{1}{2}\right)$ in space. Other interesting property of the solution is that the matrix $M_{\alpha \beta}(x)$ turns out to be real at all space points.

The next step was to study the solutions of the Dirac equation for other values of $z$. We use the same conditions to choose a basis in the space of solutions. The nice symmetry properties of the $z=0$ solutions are lost for arbitrary values of $z$, but the solution seems to show a nice smooth behaviour which makes it reliable to consider them a good approximation to the continuum limit ones.

Now, we consider the solutions of Eq. 2.16, where the left hand-side is fixed by the solutions of Eq. 2.15, as explained before. The solution is now unique, for a given choice of the direction $\mu$ and of the index $\alpha$. This is in contrast with the continuum case and is precisely due to the fact that the lattice operator has no exact zero-modes. Fortunately, we are interested in $\hat{F}_{\mu \nu}$ which is insensitive to the continuum solution chosen, hence we feel satisfied with the unique solution provided by the lattice. For all values of $z$ studied, the resulting $\Psi_{\mu}(x, z)$ obtained with this procedure look pretty smooth, suggesting that indeed we are dealing with lattice approximants to the continuum functions. Finally by use of Eqs. 2.17, 2.19 we can calculate the components of $\hat{F}_{\mu \nu}$.

The first thing to check is that the result obtained is indeed a self-dual field-strength tensor. For all the $z$ values studied this was very approximately the case. In Table 1 we show the results for some selected values of $z$. The departures from self-duality were always found to be at most of order $10^{-2}$, as for the original lattice gauge configuration. We have actually explored points in different regions of the dual torus (periodicity in $z_{\mu}$ was checked and found to work extremely well) and always got results which indicate regularity as a function of $z$.

In Fig. 3 we show a plot for the action density obtained in the plane $\left(z_{2}=1 / 2, z_{4}=\right.$ $1 / 2$ ), where a single peak appears. A total of 20 points in this plane have been generated and we used the symmetry under $\frac{\pi}{2}$ rotations around the center of this plane to increase the statistics of the surface. This symmetry has indeed been checked and works to a high level of precision.

The action density of the Nahm-transformed solution resembles very much the action density of the original configuration. When comparing one has to take into account that the centers (the actual maxima of the action density) of the original configuration do not lie exactly at a lattice point. To make a comparison which would be more precise, we made a fit to the action density of the original lattice configuration using a few Fourier components in each variable and this allowed us to interpolate the information of the original lattice configuration to points lying in between the lattice points. In Fig. 1 we compare the result of this fit (solid curve) to the actual data points obtained for the transformed field along one of the straight lines in the torus joining two centers. We emphasize that the curve is obtained by fitting the original configuration and not the Nahm-transformed one. In all the points explored we always found that the action density of the transformed field matches quite precisely the one of the original 
configuration. We proceeded to investigate systematically what is the exact relation between the original configuration and its Nahm transform by looking at the rest of the gauge invariant local quantities. These are the traces of the products of two spatial components of the electric field $\operatorname{Tr}\left(E_{i}(z) E_{j}(z)\right)$. We made a careful selection of the $z$ points to distinguish the different possibilities. The differences between the original and transformed action densities in this comparison can grow up to 5-10\%, which can be partly due to small uncertainties in the determination of the peak centers for the lattice configurations, from which the direct coordinate comparison depends. However, we found a consistent way to recognize directions by looking at the signs of the traces of crossed products of fields. Finally, we arrived at the conclusion that, up to a gauge, the relation between the original and transformed field tensors is as follows:

$$
\begin{aligned}
\hat{F}(z) & =F\left(x^{\prime}(z)\right) \\
x^{\prime}\left(z_{0}, z_{1}, z_{2}, z_{3}\right) & =\left(z_{1},-z_{0}, z_{3},-z_{2}\right)
\end{aligned}
$$

Actually, changing $z \rightarrow-z$ is also possible since the configuration is invariant under this transformation. To show an explicit example of the comparison we applied the Nahm transformation precisely at a transformed lattice point. The result is shown in Table 2, where the traces $\operatorname{Tr}\left(E_{i}(x) E_{j}(x)\right)$ are compared for two corresponding $x$ and $z$ points. To arrive to similar results for $A_{\mu}$, we should be able to compute and compare Polyakov lines. This is computationally very expensive with the present method, although some variation of it could be particularly helpful in this respect (cf. comments at the end of the concluding section).

To finish this section we want to mention another application of our numerical methods to the study of a given gauge configuration. It comes from the study of fermion zero modes in the adjoint representation of the gauge group, which can be directly adressed with our techniques. In this case there is no need to have strictly periodic gauge configurations, and we can consider also twisted background fields. The index theorem indicates that $2 N Q$ zero modes are to be found in this case. Furthermore, in contrast to the case of the fundamental representation, a new important property is expected: the supersymmetry present in the theory with adjoint fermions supplies 2 of the zero modes as supersymmetric transforms of the background (anti)self-dual gauge field [24], through

$$
\psi_{\alpha}^{A a}(x)=\frac{1}{2} \sigma_{\mu \nu}^{A B} F_{\mu \nu}^{a}(x) u_{\alpha}^{B}
$$

where $a$ is an adjoint color index in the Pauli basis, $\alpha$ an index in the space of zero modes, $A, B$ are spin indices, $u$ is the spinor parameter of the transformation, and $\sigma_{\mu \nu} \equiv \frac{\imath}{4}\left(\Gamma_{\mu} \bar{\Gamma}_{\nu}-\Gamma_{\nu} \bar{\Gamma}_{\mu}\right)$.

Our lattice computations are again able to reproduce these continuum results 18 , 19. In this case, the expected $2 N Q$-dimensional space of lowest modes actually splits into $N Q$ spaces of dimension 2, above which doubler levels appear. Each couple of 
zero modes present a geometrically distinct character, and the space related to the 'supersymmetric' zero modes can be easily identified, typically being the highest one. This allows to extract numerical approximations for the continuum field $F_{\mu \nu}$, which are expected to differ from the lattice values computed from plaquettes, as finite lattice spacing corrections enter these two quantities in completely different ways (a complete analysis of discretization errors in this new approach is still lacking). We have verified that this is indeed the case, the difference being most appreciable around absolute maxima and minima for the action density. However, the results for the gauge field coming from adjoint zero modes still compares successfully (although with larger errors) with the Nahm-transformed field when checking the correspondence between them. Further study is required in order to understand the differences in lattice corrections and to obtain a better way to compare the results consistently.

\section{Conclusions and future outlook}

In this paper we have presented a way in which one can numerically construct the Nahm transform of a self-dual Yang-Mills classical configuration discretized on the lattice. This opens the door for a more thorough study which will allow to disentangle the main features of this transformation. For example, one can study fixed points of the transformation, and relate solutions belonging to different gauge groups. This goal however requires an important computational effort and at the moment we are improving our algorithm and codes to make this a feasible task. An analysis of systematic errors is also underway. The analytic information on the transform can be of great help in suggesting the appropriate choice of initial configurations and points to study.

We have applied the Nahm transformation to a self-dual gauge field configuration built out of one carrying non-orthogonal twist. We found that in this case the transformation gives back the original configuration at a transformed point in space-time. This sort of constraint could turn out to be useful in finding the analytical solution.

To conclude, we want to comment on a point which was left out and will be developed in future publications. This is the fact that one can use techniques similar to the ones described to obtain a lattice Nahm transformation (that is, a lattice gauge configuration which is the Nahm transform of another lattice gauge configuration) by directly constructing link variables instead of continuum ones. This has a number of advantages as a parallel method; for example, Polyakov loops for the Nahm-transformed configuration can now be easily computed from lattice links.

The construction proceeds as follows. Let us first select a grid on the dual torus

$z_{\mu}=\tilde{a} n_{\mu}$, where $\tilde{a}$ is the spacing in this new lattice. The solutions $\Psi^{\alpha}(x, z)$ of Eq. 2.15 can now be computed in exactly the same way we have described. Once they are known, form the matrices:

$$
\mathcal{V}_{\mu}^{\alpha \beta}(z)=\sum_{x} \Psi^{\alpha+}(x, z) \Psi^{\beta}(x, z+\hat{\tilde{\mu}})
$$


where color and spin indices are summed over and $\hat{\tilde{\mu}}$ is a unitary vector in the $z$-lattice. These matrices transform as lattice links under a $z$-dependent change of basis in the space of zero modes, i.e. a gauge transformation in $z$-space:

$$
\begin{aligned}
\Psi^{\alpha}(x, z) & \longrightarrow \Psi^{\prime \alpha}(x, z)=\Psi^{\beta}(x, z) \Omega^{+\beta \alpha}(z) \\
\mathcal{V}_{\mu}^{\alpha \beta} & \longrightarrow \mathcal{V}_{\mu}^{\prime \alpha \beta}=\Omega^{\alpha \gamma}(z) \mathcal{V}_{\mu}^{\gamma \delta} \Omega^{+\delta \beta}(z+\hat{\tilde{\mu}})
\end{aligned}
$$

but are not unitary, as required to be identified as link variables. To extract a unitary matrix from $\mathcal{V}_{\mu}$ while keeping the transformation property we can decompose it as the product of a unitary matrix times a hermitian matrix ${ }^{1}, \mathcal{V}_{\mu}(z)=H_{\mu}(z) U_{\mu}(z)$. Finally, a $S U(Q)$ matrix has to be extracted from each $U_{\mu}$, which in general is in $U(Q)$. There is a number of ambiguities here, having to do with remaining freedoms in the 'unitarization' process, but these can in principle be dealt with by appealing to continuity arguments when going from one $z$ point to the following, once a convenient gauge has been chosen (for which the method we have described in the preceding section remains valid).

It can be seen that the naive continuum limit for the new link variables is indeed the continuum field in Eq. 2.10, although a rather complicated behaviour arises for lattice corrections, which makes it difficult to keep them controlled. This latter point seems to be the main difficulty to exploit this method. Further study, and probably use of larger lattices is required before it can be efficiently implemented.

\section{Acknowledgements}

This work was financed by the CICYT under grant AEN97-1678. An important part of the numerical calculations which are here presented have been carried on the Alphaserver8400 at the Centro de Computación Científica (UAM). We thank Álvaro Montero for help in the construction of gauge configurations and in the development of numerical methods.

\footnotetext{
${ }^{1}$ For $Q=2$ there is an easier way: the matrix $\mathcal{U}_{\mu} \equiv \mathcal{V}_{\mu}+\sigma_{2} \mathcal{V}_{\mu}^{*} \sigma_{2}$ has the property $\mathcal{U}_{\mu} \mathcal{U}_{\mu}^{+}=\operatorname{det}\left(\mathcal{U}_{\mu}\right) I$, and it is straightforward to extract a $S U(Q)$ matrix from it.
} 


\section{References}

[1] A.M. Polyakov, Phys. Lett. 59B (1975) 82.

[2] G. 't Hooft, Phys. Rev. Lett. 37 (1976) 8.

[3] A. A. Belavin, A. M. Polyakov, A. S. Schwartz and Yu. S. Tyupkin, Phys. Lett. 59B (1975) 85.

[4] M. F. Atiyah, N. J. Hitchin, V. G. Drinfeld and Y. I. Manin, Phys. Lett. 65A (1978) 185.

[5] G. K. Saviddy, Phys. Lett. 71B (1977) 133.

[6] J. Ambjørn and P. Olesen, Nuc. Phys. B170 (1977) 60.

[7] A. Gonzalez-Arroyo, Proceedings of the 1997 Peñíscola Advanced School in NonPerturbative Quantum Physics, World Scientific (1998); hep-th/9807108.

[8] G. 't Hooft, Nucl. Phys. B153 (1979) 141.

[9] G. 't Hooft, Commun. Math. Phys. 81 (1981) 267.

[10] P. van Baal, Commun. Math. Phys. 94 (1984) 397.

[11] M. García Pérez, A. González-Arroyo and B. Söderberg, Phys. Lett. 235B (1990) 117.

[12] M. García Pérez and A. González-Arroyo, J. Phys. A. 26 (1993) 2667.

[13] A. González-Arroyo and P. Martínez, Nucl. Phys. B459 (1996) 337.

[14] W. Nahm, Phys. Lett. 90B (1980) 413.

[15] P. J. Braam and P. van Baal, Commun. Math. Phys. 122 (1989) 267.

[16] Th. C. Kraan and P. van Baal, hep-th/9805168.

[17] P. van Baal, Nucl. Phys. B (Proc. Suppl.) 49 (1996) 238.

[18] M. García Pérez, A. González-Arroyo, A. Montero and C. Pena, Nucl. Phys. B (Proc. Suppl.) 63A-C (1998) 501.

[19] A. González-Arroyo, A. Montero and C. Pena, In preparation.

[20] T. Kalkreuter and H. Simma, Comput. Phys. Commun. 93 (1996) 33.

[21] H. van der Vorst, SIAM J. Sc. Stat. Comp. 13 (1992) 631.

[22] A. Frommer, V. Hanemann, B. Nöckel, Th. Lippert and K. Schilling, Int. J. Mod. Phys. C5 (1994) 1073.

[23] P. van Baal, Nucl. Phys. (Proc. Suppl.) 49 (1996) 238.

[24] E. Cohen and C. Gómez, Phys. Rev. Lett. 52 (1984) 237. 
Figure 1: Action density for the original lattice gauge configuration in the plane of the four absolute maxima. The total action has been normalized to 1 .

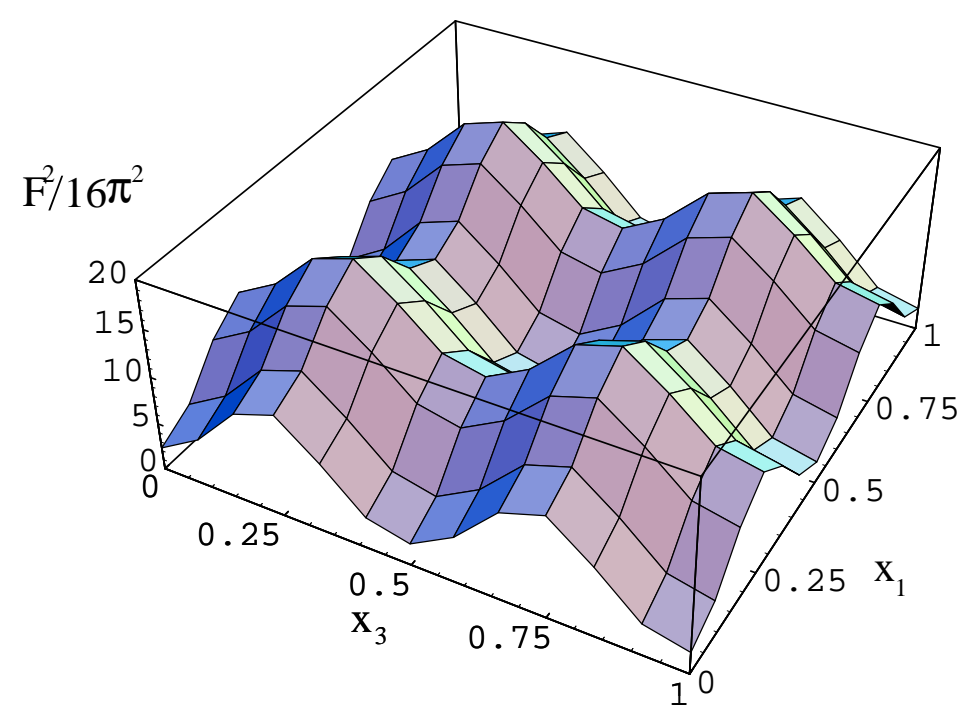


Figure 2: Invariant densities for the two orthogonal zero modes chosen for the original lattice configuration, in the plane of the four absolute maxima. Both have norm 1 in the continuum.
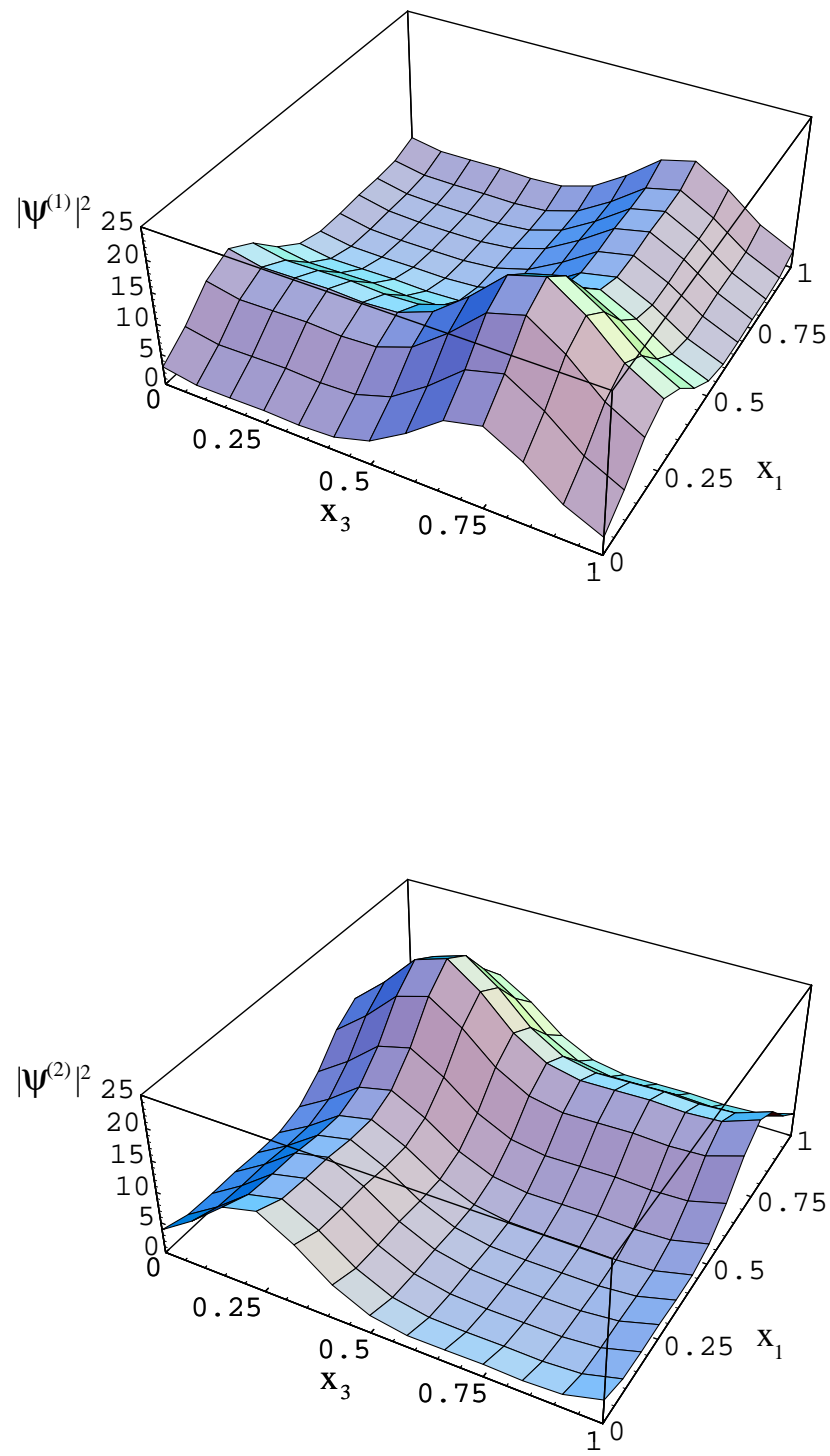
Figure 3: Action density for the transformed gauge configuration in the $\left(z_{0}=1 / 2, z_{2}=1 / 2\right)$ plane. The normalization is the same as in the original lattice configuration. The center in this plane has been displaced to the origin of coordinates for clarity, and an interpolating procedure has been used to obtain a smooth surface.

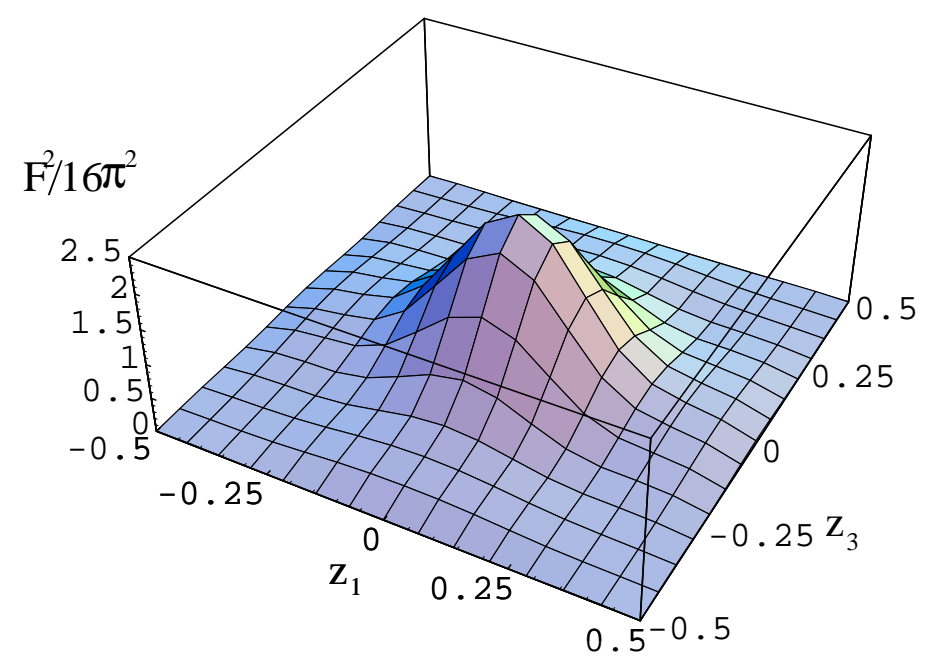


Figure 4: Comparison of action densities for the Nahm transformed field (dots) and the original lattice field (solid line) along a line with two absolute maxima. The line has been extracted from a fit to the lattice points densities. The center has been displaced to the origin of coordinates for clarity.

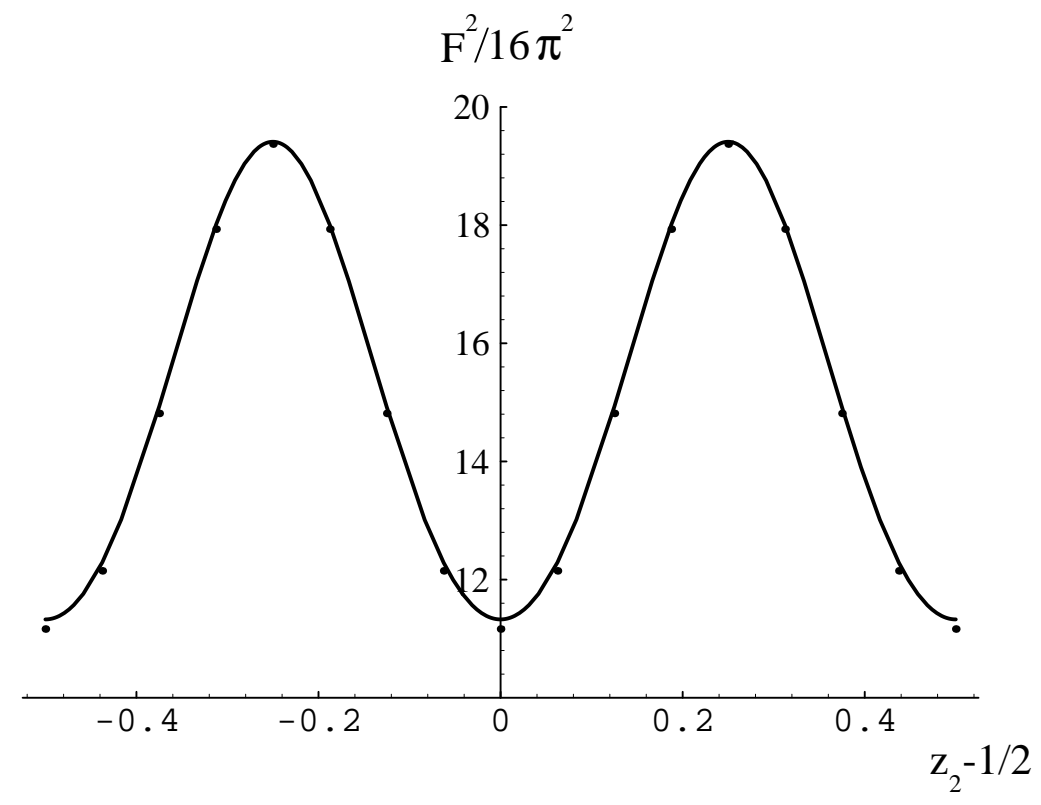


Table 1: Values for the transformed electric and magnetic gauge fields in some selected points of the $z$-space are given. The number of significative figures is dictated by the deviations from self-duality appearing in the original lattice configuration. The first point corresponds to an absolute maximum; the second, to the maximum in a plane in which $E_{1}=E_{3}=0$; the third, to a point with no special character.

\begin{tabular}{|c|c|c|}
\hline$z$-space point & $E_{1}^{a}, E_{2}^{a}, E_{3}^{a}$ & $B_{1}^{a}, B_{2}^{a}, B_{3}^{a}$ \\
\hline \hline$(1 / 2,1 / 4,1 / 2,1 / 4)$ & $(-0.049,0.012,-1.262)$ & $(-0.049,0.012,-1.262)$ \\
& $(-1.204,0.444,0.051)$ & $(-1.212,0.447,0.051)$ \\
& $(-0.436,-1.182,0.005)$ & $(-0.436,-1,182,0.005)$ \\
\hline$(1 / 2,1 / 2,1 / 2,1 / 2)$ & $(0.000,0.000,0.000)$ & $(0.000,0.000,0.000)$ \\
& $(0.218,-0.705,0.000)$ & $(0.225,-0.725,0.000)$ \\
& $(0.000,0.000,0.000)$ & $(0.000,0.000,0.000)$ \\
\hline$(3 / 4,1 / 2,1 / 2,2 / 3)$ & $(0.031,-0.001,-0.262)$ & $(0.032,-0.002,-0.262)$ \\
& $(-0.311,-0.166,-0.038)$ & $(-0.316,-0.169,-0.037)$ \\
& $(0.076,-0.287,0.010)$ & $(0.076,-0.287,0.010)$ \\
\hline
\end{tabular}

Table 2: Values for the traces of products of electric field components for corresponding $x_{0}$ and $z_{0}$ points: $x_{0}=(0.312,0.584,0.596,0.766), z_{0}=(0.584,0.688,0.766,0.404) . x_{0}$ corresponds to a lattice point, selected such that clear hierarchies among the invariants are established.

\begin{tabular}{|c|c|c|}
\hline & $x_{0}$ & $z_{0}$ \\
\hline \hline $\operatorname{Tr}\left(E_{1}(x) E_{1}(x)\right)$ & 0.418 & 0.460 \\
$\operatorname{Tr}\left(E_{2}(x) E_{2}(x)\right)$ & 0.465 & 0.488 \\
$\operatorname{Tr}\left(E_{3}(x) E_{3}(x)\right)$ & 0.383 & 0.412 \\
$\operatorname{Tr}\left(E_{1}(x) E_{2}(x)\right)$ & 0.033 & 0.031 \\
$\operatorname{Tr}\left(E_{1}(x) E_{3}(x)\right)$ & -0.027 & -0.027 \\
$\operatorname{Tr}\left(E_{2}(x) E_{3}(x)\right)$ & 0.024 & 0.025 \\
\hline
\end{tabular}

